\title{
PARTIAL FLOOR MASS ISOLATION TO CONTROL SEISMIC STRESS IN FRAMED BUILDINGS
}

\author{
M.C. PORCU \\ Department of Mechanical, Chemical and Materials Engineering, University of Cagliari, Italy.
}

\begin{abstract}
Isolating portions of the floor mass through rigid-plastic connectors may reduce the effects of strong earthquakes on framed buildings. This strategy was shown to be effective for single-storey frames, provided that a reasonably low plastic limit given to connectors and large enough portions of mass be disconnected. The stress reduction is however found to depend significantly on some interrelated parameters and on the given earthquake. By means of an analytical study and a nonlinear numerical investigation involving single-storey frames and four recorded earthquakes, the present paper gives a swift way to estimate the extent of stress reduction that can be achieved under a given earthquake, for preset values of the key parameters. Some empirical formulae are also provided to estimate the peak relative displacement that is reached by the disconnected mass.

Keywords: Floor mass isolation, inertia limiters, rigid-plastic connectors, seismic stress control.
\end{abstract}

\section{INTRODUCTION}

A successful strategy to control the seismic effects on civil structures is the base-isolation (BI) technique which introduces a flexible layer at the base of the building to drastically increase its fundamental period [1]. A different strategy may be that of isolating portions of the mass so as to lessen the inertial loads acting on the building [2-5]. Recently, partial mass isolation with nonconventional tuned mass dampers (TMDs) exploiting masses already present in the system or a combination of TMD and BI have also been proposed [6-9]. Reducing the mass, however, implies shortening of the fundamental period of vibration, which might even increase the earthquake-induced accelerations and undo the benefits of the mass reduction.

Nevertheless, the results of the numerical investigation presented in [10-12] showed that the plastic disconnection of floor mass (PDFM) approach proposed in [2] may be rather effective in controlling the seismic effects. It assumes that rigid-plastic connectors (RPCs) are inserted between structural and nonstructural floor masses. They act both as inertia limiters (disconnecting part of the mass when a pre-set level of horizontal load is reached) and as energy dissipaters (dissipating large amounts of seismic energy when they enter the plastic range). The efficacy of this method, however, was shown to depend on some interrelated parameters the influence of which is further complicated by the nonlinearity of the problem. It may thus be of practical interest to know beforehand - at least for a preliminary assessment - the extent of stress reduction that can be achieved under a given earthquake, for values of the key parameters of practical interest.

By means of an analytical study and a nonlinear numerical investigation involving single-storey frames and recorded earthquakes, this paper presents a feasible way to evaluate the stress reduction achievable for assumed values of the governing parameters directly from the earthquake response spectrum. By exploiting new results and literature data [10-12] and by investigating on the role of the key parameters, this paper also aims to provide a comprehensive assessment of the effectiveness of the PDFM approach. 
Of course, the convenience of adopting the PDFM approach has also to be assessed whilst bearing in mind the higher cost entailed by the use of RPCs that may be actually made in different ways (something like flat frictional sliders may be just one of the possible choices). Even a hysteretic or elastic-plastic behaviour might be considered for connectors. In any case, an appropriate design will be required to sustain the detached mass, tolerate its relative displacements and re-centre it. Rigid diaphragms or secondary beams may be needed to hold the disconnected masses (some ideas may be borrowed from $[4,13]$ ). The possibility to substitute connectors as well as the design of installations to absorb relative displacements, at least for medium earthquakes, should also be considered. These problems (most of which are habitually dealt with in earthquake engineering practice) are not examined in this paper, the main aim of which is to evaluate whether and when the PDFM approach might be effectively adopted in practice.

\section{ANALYTICAL MODEL}

A shear-type single-storey frame of mass $m$, lateral stiffness $k$ and damping ratio $\xi$ is considered in Fig. 1a (Frame A). Under a given ground acceleration, $\ddot{u}_{g}(t)$, the motion equation of this single-degree-of-freedom (SDOF) system is

$$
\ddot{u}+\frac{4 \pi}{T} \xi \dot{u}+\frac{4 \pi^{2}}{T^{2}} u=-\ddot{u}_{g}(t) .
$$

Here $u, \dot{u}$ and $\ddot{u}$ are displacement, velocity and acceleration of mass $m$, respectively, while $T=2 \pi \sqrt{\mathrm{m} / \mathrm{k}}$ is the natural period of the system. The maximum stress reached by pillars of Frame $A$ during ground motion can be obtained by applying the following equivalent horizontal static force to mass $m$ :

$$
F_{\text {eqA }}=k u_{E_{-} \max }(T, \xi) \cong m a_{E_{-} \max }(T, \xi)
$$

In eqn (2), $u_{E-\max }(T, \xi)$ is the peak elastic displacement reached by mass $m$ during the given earthquake, while $a_{E_{-} \max }$ is the pseudo-acceleration given by [14]

$$
a_{E_{-} \max }=\frac{4 \pi^{2}}{T^{2}} u_{E_{-} \max }
$$

The 2DOF system referred to as Frame B in Fig. 1b is obtained from Frame A by sharing mass $m$ into two portions, say $m_{1}$ and $m_{2}$. The upper mass $m_{2}$ is connected to lower mass $m_{1}$

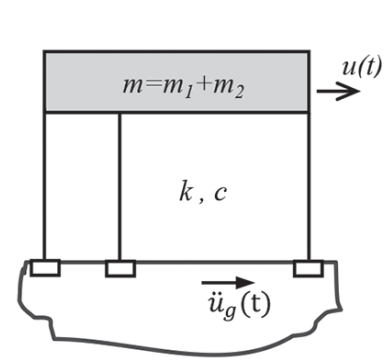

(a) Frame A

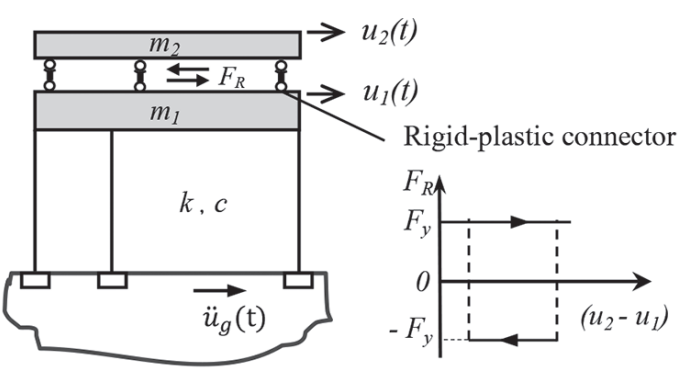

(b) Frame B

(c) Connectors' behaviour

Figure 1: (a) Elastic SDOF frame; (b) inelastic 2DOF system; (c) rigid-perfectly-plastic behaviour of connectors. 
through rigid-perfectly-plastic devices which are supposed to behave symmetrically in both directions, see Fig. 1c. The motion equations of this inelastic system can be written as $[2,10,11]$

$$
\begin{gathered}
\text { Elastic range: if }\left|\ddot{u}_{2}^{t}\right|<a_{y} \text { or if }\left|\ddot{u}_{2}^{t}\right|=a_{y} \text { and } \operatorname{sgn}\left(\dot{u}_{2}\right) \neq \operatorname{sgn}\left(u_{2}\right) \\
\left\{\begin{array}{c}
\ddot{u}_{1}+\frac{4 \pi}{T} \xi \dot{u}_{1}+\frac{4 \pi^{2}}{T^{2}} u_{1}=-\ddot{u}_{g}(t), \\
u_{2}=u_{1} .
\end{array}\right. \\
\text { Plastic range: if }\left|\ddot{u}_{2}^{t}\right|=a_{y} \text { and } \operatorname{sgn}\left(\dot{u}_{2}\right)=\operatorname{sgn}\left(u_{2}\right) . \\
\left\{\begin{array}{c}
\left(1-\rho_{m}\right) \ddot{u}_{1}+\frac{4 \pi}{T} \xi \dot{u}_{1}+\frac{4 \pi^{2}}{T^{2}} u_{1}-\operatorname{sgn}\left(u_{2}-u_{1}\right) \rho_{m} \rho_{y} a_{E_{-} \max }=\left(p_{m}-1\right) \ddot{u}_{g}(t), \\
\ddot{u}_{2}+\operatorname{sgn}\left(u_{2}-u_{1}\right) \rho_{y} a_{E_{-} \max }=-\ddot{u}_{g}(t) .
\end{array}\right.
\end{gathered}
$$

Here, $\ddot{u}_{2}^{t}$ is the total acceleration (inertial) of mass $m_{2} ; \rho_{m}=m_{2} / m$ is the percentage of disconnected mass while $\rho_{y}=a_{y} / a_{E_{-} \max }$ quantifies the plastic involvement of the RPCs during the earthquake; $a_{y}=F_{y} / m_{2}$ is the total acceleration of mass $m_{2}$ at yield (yield acceleration) which is also the maximum acceleration reachable by mass $m_{2}$ due to yield load $F_{y}$. Equations (4a) and (4b) show that the response of Frame $B$ to a given earthquake depends on four parameters: $T, \xi, \rho_{m}$ and $\rho_{y}$. For a given earthquake, the peak elastic acceleration $a_{E \_ \text {max }}$ is in fact a known quantity once the response spectrum is given. The maximum stress reached by pillars of Frame $B$ can be obtained by applying the following static force:

$$
F_{e q B}=k u_{1 \_\max }\left(T, \xi, \rho_{m}, \rho_{y}\right) .
$$

\section{STRESS REDUCTION AND PEAK RELATIVE DISPLACEMENTS}

By referring to the frames in Fig. 1 and to eqns (2) and (5), the effectiveness of the PDFM approach can be assessed through the following ratio [2]:

$$
\Delta_{s}=\Delta_{s}\left(T, \xi, \rho_{m}, \rho_{y}\right)=\frac{F_{e q B}-F_{e q A}}{F_{e q A}}=\frac{u_{1_{-} \max }}{u_{E_{-} \max }}-1 .
$$

$\Delta_{s}$ can be referred to as the stress reduction factor, since the seismic stress on columns of both Frame $A$ and Frame $B$ is proportional to the static force applied. Whenever $u_{1 \_\max }<u_{E_{-} \max }, \Delta_{S}$ assumes a negative value (lower than one) which indicates a stress reduction. The lower the displacement ratio, $u_{1 \_ \text {max }} / u_{E_{-} \max }$ the higher the stress reduction and, accordingly, the more effective the PDFM strategy is. Fig. 2a provides the displacements $u(t)$ of Frame $A$ and $u_{1}(t)$ of Frame $B$, as obtained from a numerical analysis for a given ground motion and assigned values of, T, $\xi, \rho_{m}$ and $\rho_{y}$ (given in the figure). A reduction in the peak displacement (and, thus, in the stress) of about $46 \%$ may be achieved in this case. 
(a)

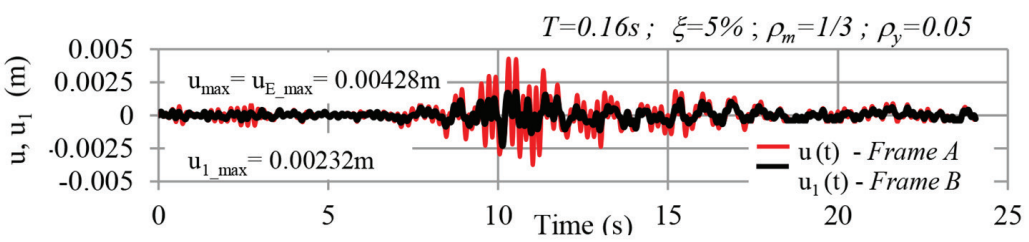

(b)

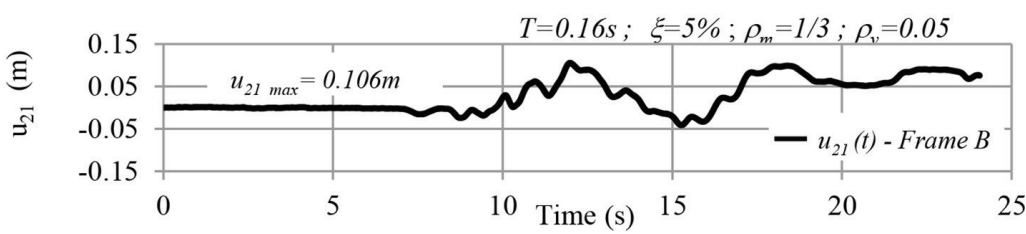

Figure 2: (a) Comparison of displacements $u(t)$ and $u_{1}(t)$ under the Kocaeli (Turkey, ATS000, 1999) earthquake; (b) relative displacement $u_{21}(t)$.

It should be considered, however, that the price to pay for high stress reduction could be large peak relative displacements of the disconnected mass, that is,

$$
u_{21 \_\max }\left(T, \xi, \rho_{m}, \rho_{y}\right)=\left|\max \left[u_{2}(t)-u_{1}(t)\right]\right| .
$$

The relative displacement $u_{21}(t)$ relevant to the instance considered in Fig. 2a is plotted in Fig. 2b. A peak displacement of about $10 \mathrm{~cm}$ is reached in this case.

\section{STRESS REDUCTION FROM THE ELASTIC RESPONSE SPECTRUM}

To obtain $\Delta_{S}$ from eqn (6), both $u_{1 \_\max }$ and $u_{E_{-} \max }$ must be derived in advance. While $u_{E_{-} \max }$ can quickly be taken from the earthquake response spectrum, a time-consuming integration of nonlinear eqns (4a)-(4b), should be carried out to calculate $u_{1 \_}$max . A swift - but approximate - way to obtain $u_{1 \_ \text {max }}$ can be found by assuming that, during a given earthquake, the peak displacement $u_{1_{-} \max }$ is reached while the system is in the plastic range, that is, when eqns $(4 \mathrm{~b})_{1,2}$ apply. As the term $\operatorname{sgn}\left(u_{2}-u_{1}\right)$ appears in both of eqn $(4 \mathrm{~b})_{1}$ and eqn (4b) $)_{2}$, eqns $(4 \mathrm{~b})_{1,2}$ are known as coupled equations. Should the case that a positive (or a negative) sign be assumed in place of $\operatorname{sgn}(\cdot)$, they would become uncoupled and, above all, linear. This statement may be exploited to predict $u_{1_{-} \max }$ under a given earthquake, through the linear differential equation:

$$
\left(1-\rho_{m}\right) \ddot{u}_{1}+\frac{4 \pi}{T} \xi \dot{u}_{1}+\frac{4 \pi^{2}}{T^{2}} u_{1}=\left(\rho_{m}-1\right) \ddot{u}_{g}(t)+\rho_{m} \rho_{y} a_{E_{-} \max } .
$$

Equation (8) is obtained from eqn (4b) by assuming a positive value for $\operatorname{sgn}($.$) and by$ shifting the constant term to the right-hand side. The choice of assuming a negative sign would lead to the same results, since the peak absolute displacements are to be considered for the effect combination. It can be now noted that eqn (8) is the motion equation of an elastic oscillator of period $T_{1}$ and damping ratio $\xi_{1}$ given by

$$
T_{1}=2 \pi \sqrt{\frac{m_{1}}{k}}=T \sqrt{\left(1-\rho_{m}\right)} ; \quad \xi=\frac{\xi}{\sqrt{\left(1-\rho_{m}\right)}} .
$$


In view of eqns $(9)_{1}$ and $(9)_{2}$, eqn (8) can also be put in the form:

$$
\ddot{u}_{1}+\frac{4 \pi}{T_{1}} \xi_{1} \dot{u}_{1}+\frac{4 \pi^{2}}{T_{1}^{2}} u_{1}=-\ddot{u}_{g}(t)+\frac{\rho_{m}}{\left(1-\rho_{m}\right)} \rho_{y} a_{E_{-} \max } .
$$

Due to the linearity, the solution of eqn (10) may be found by superposing the solutions of the following two equations:

$$
\begin{gathered}
\ddot{u}_{1}+\frac{4 \pi}{T_{1}} \xi_{1} \dot{u}_{1}+\frac{4 \pi^{2}}{T_{1}^{2}} u_{1}=-\ddot{u}_{g}(t) \\
\ddot{u}_{1}+\frac{4 \pi}{T_{1}} \xi_{1} \dot{u}_{1}+\frac{4 \pi^{2}}{T_{1}^{2}} u_{1}=\frac{\rho_{m}}{\left(1-\rho_{m}\right)} \rho_{y} a_{E_{-} \max .}
\end{gathered}
$$

Equation (11) is the motion equation of an oscillator of period $T_{1}$ and damping ratio $\xi_{1}$ under the ground acceleration $\ddot{\mathrm{u}}_{g}(t)$. Its integration leads to the calculation of $u_{1 E_{-} \max }$, the value of which can also be taken directly from the earthquake response spectrum. Equation (12) is the motion equation of the same oscillator subjected to a step load. Also in view of eqn (3), the peak response obtained from eqn (12) for at rest initial conditions is

$$
u_{1 \text { STEP }}\left(\rho_{m}, \rho_{y}, \xi, T_{1}\right)=\frac{\rho_{m}}{\left(1-\rho_{m}\right)} \rho_{y} u_{E_{-} \max }\left(1+e \frac{-\xi \pi}{\sqrt{\rho_{m}-\xi^{2}}}\right)
$$

An estimate of the actual inelastic peak displacement $u_{1_{-} \max }$, say $\bar{u}_{1_{-} \max }$, may be obtained by combining the two peak responses $u_{1 E_{-} \max }$ and $u_{1 S T E P}$ through the square root of sum of squares rule, i.e. $\bar{u}_{1 \_ \text {max }}=\sqrt{u_{1 E_{-} \max }^{2}}+u_{1 \text { STEP }}^{2}$. Thus, an estimate of $\Delta_{S}$ as defined by eqn (6) can be finally obtained as

$$
\bar{\Delta}_{S}=\frac{\bar{u}_{1 \_ \text {max }}}{u_{E_{-} \max }}-1=\sqrt{\left(\frac{u_{1 E_{-} \max }}{u_{E_{-} \max }}\right)^{2}+\frac{\rho_{m}^{2} \rho_{y}^{2}}{\left(1-\rho_{m}\right)^{2}}\left(1+e^{\frac{-\xi \pi}{\sqrt{\rho_{m}-\xi^{2}}}}\right)^{2}}-1 .
$$

To check the reliability of eqn (14), the values of $\bar{\Delta}_{S}$ (estimated) were compared to the actual values $\Delta_{S}$ (calculated) obtained from rigorous numerical analyses for different sets of parameters under four recorded earthquakes. The results collected in Fig. 3 show that eqn (14) provides on the whole a very good estimate of the actual value of $\Delta_{S}$ (mean errors of about $11-15 \%)$. Accelerograms, elastic response spectra and rigid-plastic pseudo-spectra [15] of the considered earthquakes are displayed in Fig. 4. Figures 5a-5b show that practical and suitable ranges of $\rho_{y}$ and $\rho_{m}$ are typically $0.05 \leq \rho_{y} \leq 0.25$ and $0.2 \leq \rho_{m} \leq 0.5$. A sort of 'knee' exhibited by the curves in Fig. 5a highlights that disconnecting more than a half of the total mass is not convenient. On the other hand, Fig. $5 \mathrm{~b}$ shows that a stress reduction even up to $50 \%$ can be obtained for $\rho_{y} \leq 0.25$, provided that at least one-third of the mass is disconnected. Whatever the earthquake, however, there is no convenience in setting the value of $\rho_{y}$ lower than 0.05 since it would actually imply an increase in relative displacements (due to the drop in plastic energy dissipation) against a slight increase in stress reduction (see Figs 5a and $5 \mathrm{c}$ ). The stress reduction also depends on the earthquake and the natural period $T$ (see Fig. 3), while $\xi$ has generally a quite small effect on the effectiveness of the method [10]. 

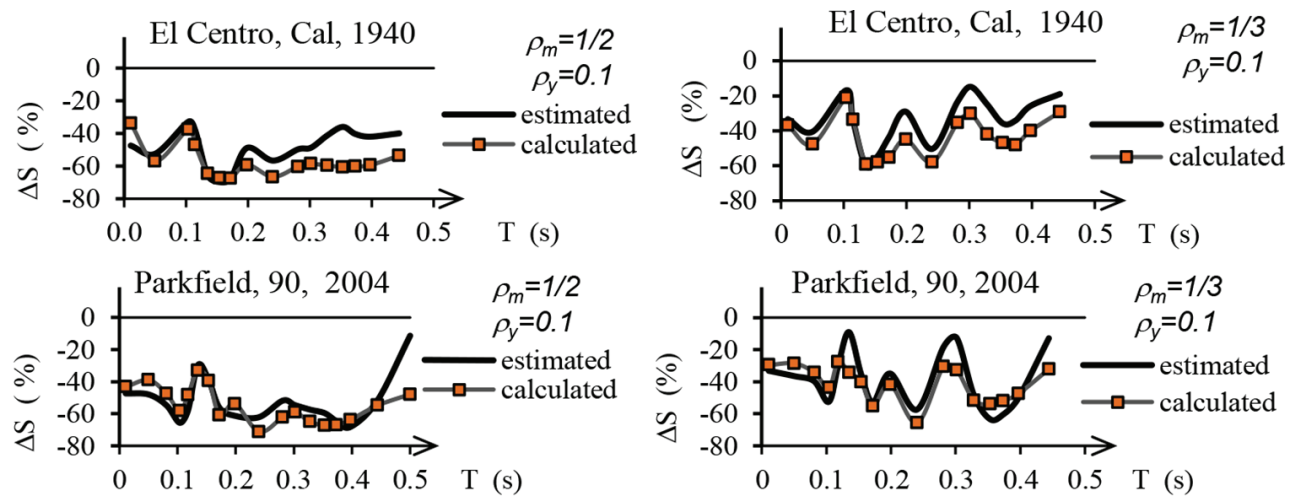

Chi-Chi, Taiwan, CHY041 N, $1999 \rho_{m}=1 / 2$

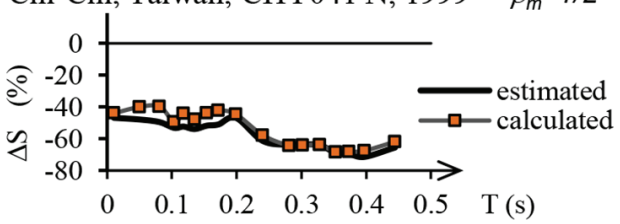

Chi-Chi, Taiwan, CHY041 N, 1999
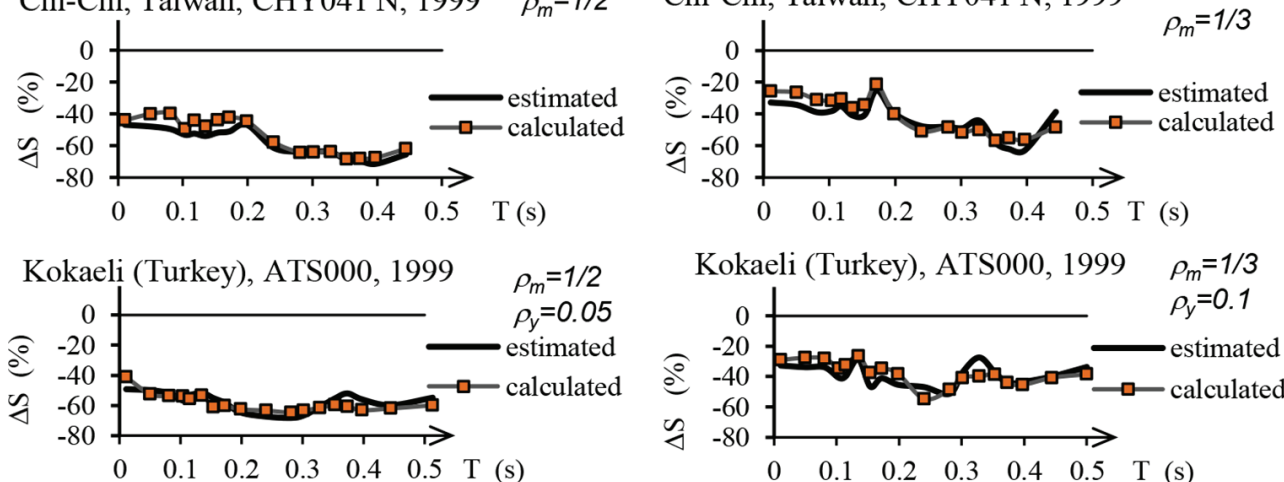

Kokaeli (Turkey), ATS000, $1999 \rho_{m}=1 / 3$

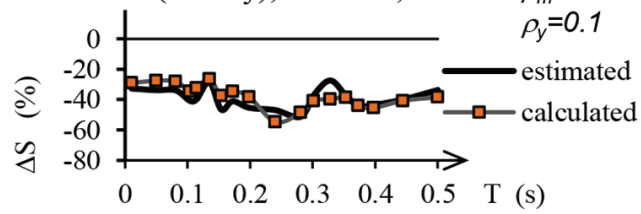

Figure 3: Calculated and estimated values of the stress reduction factor $(\xi=5 \%)$.
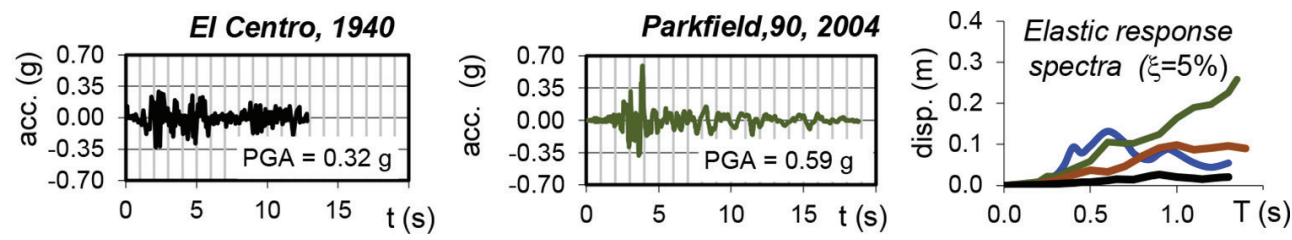

Chi Chi, Taiwan, CHY041, 1999
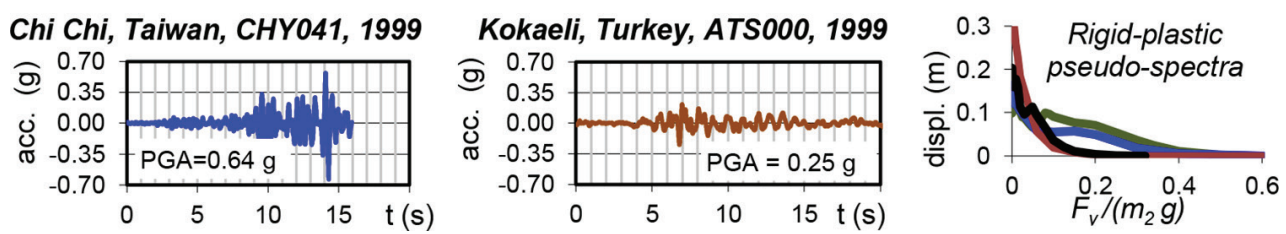

Figure 4: Accelerograms and spectra of the earthquakes considered in the analysis. 


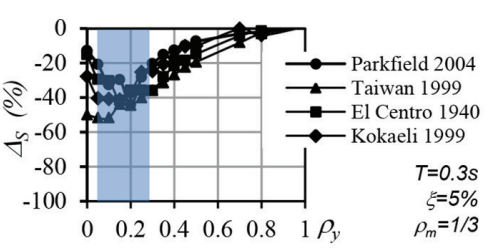

(a)

(c)

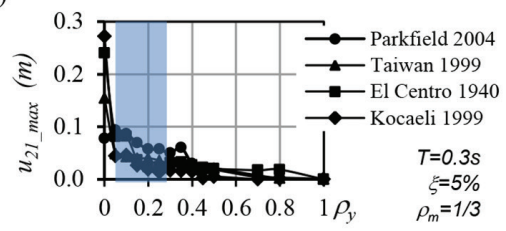

(b)
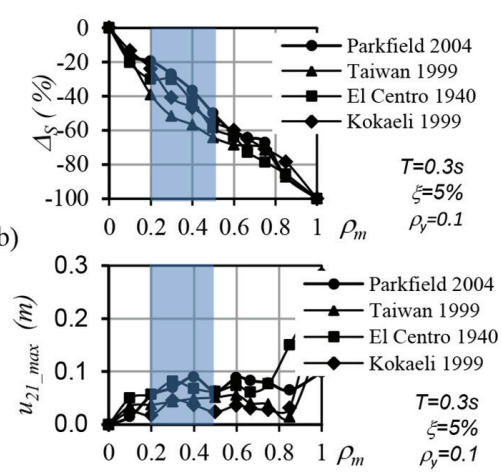

Figure 5: Role of key parameters on stress control (in grey ranges of practical interest).

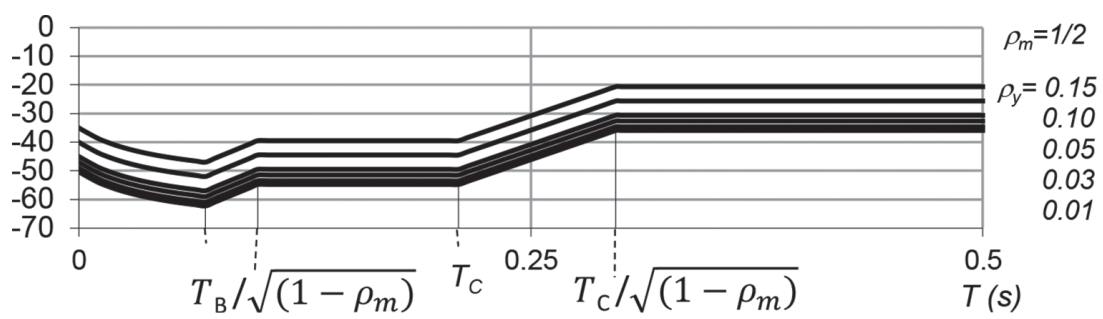

Figure 6: Stress reduction obtained from the EC8 elastic response spectrum.

Equation (14) can even be exploited to estimate the stress reduction from the design response spectrum. To this purpose, the ratio between the design peak pseudo-accelerations, namely, $R_{a}=S_{a 1}\left(T_{1}, \xi_{1}\right) / S_{a}(T, \xi)=m u_{1 E_{-} \max } / m_{1} u_{E_{-} \max }$ may be considered. As a function of this ratio, eqn (14) becomes

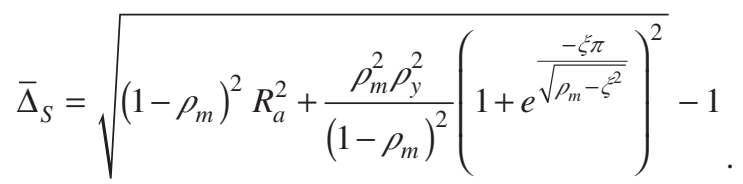

With reference to Eurocode 8 (EC8), the value of $R_{a}$ was obtained in [10] for the different ranges of the spectral characteristic periods. An instance of the stress reduction achievable from the EC8 response spectrum is given in Fig. 6.

\section{RELATIVE DISPLACEMENT FROM THE RIGID-PLASTIC PSEUDO-SPECTRUM}

The following semi-empirical formulas were derived in [12] to estimate the peak relative displacement $u_{21 \_} \max$

$$
\begin{gathered}
\text { for } T<0.3 s \quad u_{21 \_\max } \cong u_{\max }^{R P}\left(a_{y}\right), \\
\text { for } T \geq 0.3 s \quad u_{21 \_\max } \cong u_{\max }^{R P}\left(\frac{a_{y}}{2}\right) .
\end{gathered}
$$



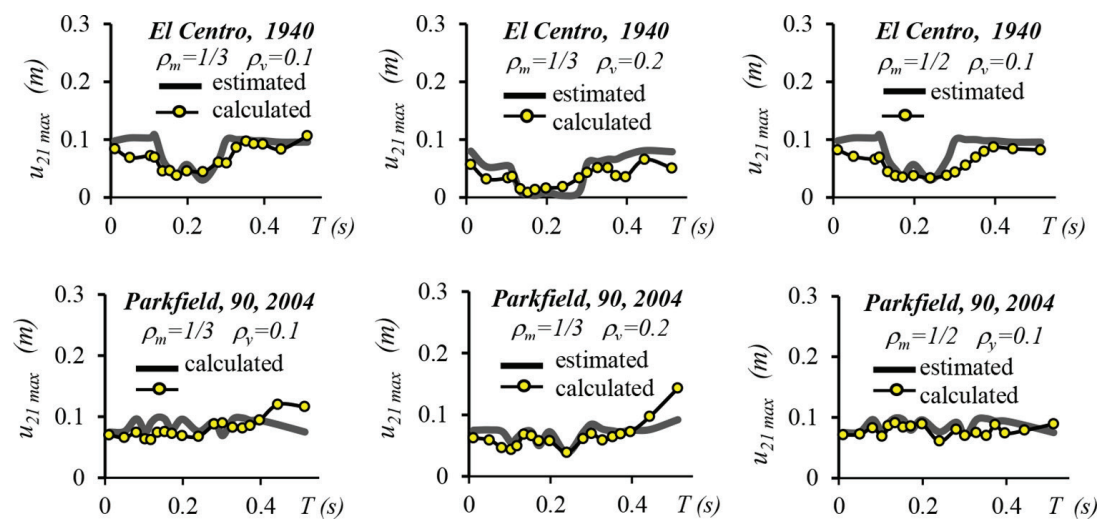

Figure 7: Calculated and estimated values of the peak relative displacement $(\xi=5 \%)$.

Here $u_{\max }^{R P}\left(a_{y}\right)$ is the peak displacement of a rigid-perfectly-plastic oscillator of mass $m_{2}$ and yield load $F_{y}$, cf. [15-17]. The value of $u_{\max }^{R P}\left(a_{y}\right)$ may be taken from the rigid-plastic pseudo-spectrum (see Fig. 3), which provides the peak plastic displacement of a rigidperfectly-plastic oscillator under a given earthquake as a function of the yield acceleration $a_{y}=F_{y} / m_{2}$ [15]. The comparison between the calculated values obtained from a complete numerical analysis and the estimated values derived from eqns (16)a,b shows that the rigidplastic estimate is very good. Figure 7 shows, in particular, that rather similar calculated values are obtained under a given earthquake for the same $\rho_{y}$ but different $\rho_{m}$, as also shown in Fig. 5d. Comparatively, low values of $u_{21}$ max are typically found in the instances presented in Figs $5 \mathrm{c}, 5 \mathrm{~d}$ and 7 , not exceeding $10 \mathrm{~cm}$ in the ranges of practical interest.

\section{CONCLUSIONS}

The results presented here show that the PDFM strategy may reduce significantly the effects of strong ground motions on framed buildings (up to 40\%-60\%), although involving comparatively small relative displacements of the disconnected mass (about $10 \mathrm{~cm}$ in the considered instances). By referring for simplicity to single storey frames, this paper presented an approximate - but quick and reliable - procedure that leads to estimate beforehand the stress reduction for any set of governing parameters and under any earthquake, provided that the earthquake response spectrum is available. An analytical study and a wide numerical investigation involving linear and nonlinear systems under different recorded earthquakes have been carried out to derive and assess this procedure. Empirical formulae are also provided to estimate the relative displacements of the disconnected mass through the rigid-plastic pseudo-spectrum. The role of the key parameters and the ranges of practical interest have also been discussed in the paper, while new results and literature data have been gathered together to provide a more comprehensive assessment of the method. Although further investigations are certainly required to definitely assess the effectiveness of the PDFM approach, the results presented in this paper highlight the potential of this partial mass isolation technique (which is expected to be effective also for multi-storey buildings) thereby encouraging more in-depth investigations with a view to its practical application. 


\section{REFERENCES}

[1] Warn, G.P. \& Ryan, K.L., A review of seismic isolation for buildings: historical development and research needs. Buildings, 2(3), pp. 300-25, 2012.

[2] Paglietti, A. \& Porcu, M.C., Controlling dynamic stress through plastic limiters. Proc. of the 1st European Conference on Structural Control, eds. A. Baratta. and J. Rodelar, Barcelona, Spain, World Scientific Publishing, Singapore, pp. 483-90, 1996.

[3] Ziyaeifar, M. \& Noguchi, H., Partial mass isolation in tall buildings. Earthquake Engineering \& Structural Dynamics, 27, pp. 49-65, 1998.

[4] Villaverde, R., Aguirre M. \& Hamilton, C., Aseismic roof isolation system built with steel oval elements: exploratory study. Earthquake Spectra, 21(1), pp. 225-41, 2005.

[5] Tian, Z.C., Qian, J.R. \& Zhang, L.M., Slide roof system for dynamic response reduction. Earthquake Engineering \& Structural Dynamics, 37(4), pp. 647-58, 2008.

[6] Anajafi, H. \& Medina, R.A., Comparison of the seismic performance of a partial mass isolation technique with conventional TMD and base-isolation systems under broad-band and narrow-band excitations. Engineering Structures, 158, pp. 110-123, 2018.

[7] Reggio, A. \& De Angelis, M., Optimal energy-based seismic design of non-conventional Tuned Mass Damper (TMD) implemented via inter story isolation. Earthquake Engineering \& Structural Dynamics, 44(10), pp. 1623-42, 2015.

[8] Hashimoto, T., Fujita, K., Tsuji, M. \& Takewaki, I., Innovative base-isolated building with large mass-ratio TMD at basement. Int. J. Future Cities Environ. 1, p. 9, 2015.

[9] De Domenico, D. \& Ricciardi, G., An enhanced base isolation system equipped with optimal tuned mass damper inerter (TMDI). Earthquake Engineering \& Structural Dynamics, 47, pp. 1169-1192, 2018.

[10] Porcu, M.C., Plastic disconnection of story masses for seismic stress control. In Earthquake Resistant Engineering Structures X, 152, WIT Press, 2015.

[11] Porcu, M.C., Numerical assessment of a stress control method based on rigid-plastic inertia-limiters. Proceedings of the 14th Int. Conference on Civil, Structural and Environmental Eng. Computing, Civil-Comp Press, Stirlingshire, UK, Paper 42, 2013.

[12] Porcu, M.C., Reducing seismic stress on buildings through inertia limiters at floor level, earthquake resist. Engineering Structures, IX, 132-333, 2013.

[13] Xiang, P. \& Nishitani, A., Seismic vibration control of building structures with multiple tuned mass damper floors integrated. Earthquake Engineering \& Structural Dynamics, 43(6), pp. 909-925, 2014.

[14] Chopra, A.K., Dynamics of Structures, Prentice-Hall, New Jersey, USA, 2001.

[15] Paglietti, A. \& Porcu, M.C., Rigid-plastic approximation to predict plastic motion under strong earthquakes. Earthquake Engineering \& Structural Dynamics, 30, pp. 115-126, 2001.

[16] Porcu, M.C. \& Carta, G., A better rigid-plastic estimate for earthquake-induced plastic displacements. International Journal of Safety and Security Engineering, 2(2), pp. 184-196, 2012.

[17] Porcu, M.C. \& Carta, G., Evaluating a rigid-plastic method to estimate the earthquake ductility demand on structures. In Earthquake Resistant Engineering Structures VIII, 120, Wit Press, pp. 261-271, 2011. 